\title{
Range compensation for backscattering measurements in the difference-frequency nearfield of a parametric sonar
}

\author{
Kenneth G. Foote ${ }^{a)}$ \\ Woods Hole Oceanographic Institution, Woods Hole, Massachusetts 02543
}

(Received 12 August 2011; revised 27 January 2012; accepted 30 January 2012)

\begin{abstract}
Measurement of acoustic backscattering properties of targets requires removal of the range dependence of echoes. This process is called range compensation. For conventional sonars making measurements in the transducer farfield, the compensation removes effects of geometrical spreading and absorption. For parametric sonars consisting of a parametric acoustic transmitter and a conventional-sonar receiver, two additional range dependences require compensation when making measurements in the nonlinearly generated difference-frequency nearfield: an apparently increasing source level and a changing beamwidth. General expressions are derived for range compensation functions in the difference-frequency nearfield of parametric sonars. These are evaluated numerically for a parametric sonar whose difference-frequency band, effectively $1-6 \mathrm{kHz}$, is being used to observe Atlantic herring (Clupea harengus) in situ. Range compensation functions for this sonar are compared with corresponding functions for conventional sonars for the cases of single and multiple scatterers. Dependences of these range compensation functions on the parametric sonar transducer shape, size, acoustic power density, and hydrography are investigated. Parametric range compensation functions, when applied with calibration data, will enable difference-frequency echoes to be expressed in physical units of volume backscattering, and backscattering spectra, including fish-swimbladder-resonances, to be analyzed.
\end{abstract}

(C) 2012 Acoustical Society of America. [http://dx.doi.org/10.1121/1.3688505]

PACS number(s): 43.30.Lz, 43.30.Sf, 43.60.Fg, 43.80.Jz [ADP]

Pages: $3698-3709$

\section{INTRODUCTION}

In 2008 and 2009, extended aggregations and compact schools of Atlantic herring (Clupea harengus) were observed in situ in the water column by parametric sonar. ${ }^{1}$ The particular parametric sonar ${ }^{2}$ was a commercial realization of the parametric acoustic array in water, ${ }^{3}$ optimized for subbottom profiling. The lowness of its nonlinearly generated difference frequencies, in the effective band $1-6 \mathrm{kHz}$, opens the possibility of measuring the swimbladder resonance frequency, hence inferring the herring size or size distribution. ${ }^{4,5}$ At the same time, other quantification of the herring can be entertained; for example, to determine the numerical density of aggregations by echo integration ${ }^{6,7}$ and, in conjunction with the concurrent use of other sonars, to measure possible behavioral reactions to the measurement process. ${ }^{8}$

Prerequisites for measuring acoustic backscattering properties of targets are range compensation and calibration. Range compensation is important for removing the range dependence of echoes, enabling the acoustic backscattering properties of targets to be determined to within a scaling factor, known as the calibration constant or factor at a fixed range. ${ }^{9}$ Calibration is a separate, independent matter. Both range compensation and calibration are sufficiently complicated to warrant separate treatment. The subject of this paper, range compensation, specifically treats the nearfield of parametric sonars. However, it can also be applied to conventional sonars so as to extend their ordinary operating range into the transducer nearfield. ${ }^{10}$

\footnotetext{
a) Author to whom correspondence should be addressed. Electronic mail: kfoote@whoi.edu
}

There is a fundamental difference in the nature of range compensation with respect to conventional and parametric sonars. This is elaborated in the next two subsections.

\section{A. Range compensation for conventional sonars}

The intensity of sonar echoes generally decreases with increasing target range in the transducer farfield due to the simple effects of geometrical spreading and absorption. The farfield is the region where the transmit pressure amplitude is inversely proportional to range. The relationship of transmit pressure amplitude and range is usually quite complicated in the transducer nearfield, where interference effects can be quite pronounced, being strongest for the circular shape. ${ }^{11}$ There are entirely pragmatic reasons for marking the transition to the farfield, which is asymptotic. The Rayleigh distance measures this transition; at greater ranges, the inverse relationship of transmit pressure amplitude and range can be assumed to apply with high accuracy. For a planar circular transducer of radius $a$, the Rayleigh distance is $2 a^{2} / \lambda$, where $\lambda$ is the acoustic wavelength. This distance measure has been generalized to other projector shapes. In the case of linear arrays of length $L$, the Rayleigh distance is $L^{2} /(2 \lambda)$, although the more conservative measure $L^{2} / \lambda$ is sometimes used. ${ }^{11}$ For other planar shapes, the same farfield distance measure can be used as for the linear array, but where $L$ is interpreted as the maximum linear dimension.

The echo intensity from a small target in the transducer farfield will vary as $\exp (-4 \beta r) / r^{4}$, where $\beta$ is the amplitude absorption coefficient, and the echo intensity from a layer of uniformly, randomly distributed targets will vary as $\exp (-4 \beta r) / r^{2}$. To remove the range dependence, the echo 
intensity is generally multiplied by the respective inverse, depending on whether the object of the measurement is the backscattering cross section of a single target or the volume backscattering coefficient of a distribution of targets. Two range compensation functions are thus described. Historically, these would have been applied electrically or electronically in real time in the receiver as the echo data were being received. The application was called time-varied gain (TVG) ${ }^{12-14}$ since $r=c t / 2$, where $c$ is the speed of sound and $t$ is the echo time measured from the start of transmission. It has received considerable attention in applications of acoustics in fisheries research because of the sensitivity of acoustic estimates of the numerical density of fish to this form of signal amplification. Currently, at least in some systems, range compensation is applied or corrected during the postprocessing of echo data, with advantages from using sound speed profiles that more accurately reflect the hydrographic conditions of data collection, as foreseen in the development of the Bergen Echo Integrator. ${ }^{15}$ There are other advantages too, for example, avoiding introducing nonlinearities into the echo processing by applying an increasing gain function over finite-duration echo signals, also discussed below in Sec. VI D.

The simple functions described here do not generally apply in the transducer nearfield, and making quantitative measurements in this region is correspondingly difficult. Not only does the transmitted pressure amplitude not vary simply as $1 / r$, but the transducer directionality also varies with $r$. With many scientific echo sounders this is not an ordinary operational matter, because the transducer nearfield is so short. For the Simrad EK60 scientific echo sounder ${ }^{16}$ operating at discrete frequencies from 18 to $200 \mathrm{kHz}$, for example, the farfield is typically $1-3 \mathrm{~m}$, decreasing with increasing frequency. For most applications to fish and zooplankton, typical operating ranges at these frequencies are of order $5 \mathrm{~m}$ or more. Echoes may not even be recorded at shorter ranges because of interference by the transmit signal and associated reverberation from structures near the transducer.

\section{B. Approach for parametric sonar}

In the special case of the parametric acoustic array ${ }^{3}$ as a transmitter of secondary, difference-frequency radiation, nearfield effects cannot generally be ignored. For commercial parametric sonars designed and used for sub-bottom profiling, with parametric transmitter and conventional-sonar receiver, as in the present case, ${ }^{2}$ and with difference frequencies in the low-kilohertz band, the difference-frequency nearfield extends over many hundreds of meters. For potential applications to fish, for example, this is the very region where measurements are wanted. It is appreciated that the difference-frequency field is generated by the nonlinear interaction of collinear, primary-frequency fields throughout the volume in front of the transducer, in a so-called virtual endfire array. The difference-frequency field is literally forming, or building up, with increasing range before diminishing at a rate slower than $1 / r$ until the farfield is reached. At the same time, the beam of the generated difference-frequency field is changing with range. For the particular parametric sonar of interest, ${ }^{2}$ the transmit beam sharpens with increasing range, that is, its beamwidth decreases with increasing range, as with the parametric transmitting arrays measured by Muir and Willette. ${ }^{17}$

If such a parametric sonar is to be used quantitatively in the water column, then nearfield effects must be addressed. It is the initial aim of this paper to derive general expressions for the range compensation functions of arbitrary parametric sonars for quantifying both single targets and distributions, or aggregations, of targets. Since the details of the nearfield of parametric sonars depend on the particular transducer dimensions and operating characteristics, the expressions are evaluated for the parametric sonar of immediate interest.

Following the development of theory, the related technical specifications of the parametric sonar of particular interest are given. To derive required nearfield characteristics, the CONVOL5 computer code ${ }^{18}$ is used to model transmit beam patterns and on-axis sound pressure levels, but after testing of the code against historical measurements of other parametric sonars. The equivalent beamwidth, ${ }^{19}$ or equivalent beam angle ${ }^{14}$ is derived from the modeled transmit and receive beam patterns. The range compensation functions are then numerically evaluated. Dependences of these on the transducer shape, hydrography, acoustic power density, and transducer area are also investigated.

\section{THEORY}

\section{A. Range compensation for conventional sonars and ordinary farfield applications}

Ordinarily, sonars, including echo sounders, are used to make measurements in the farfield of collocated transmitting and receiving transducers. By definition, the transmit field amplitude in this region decreases inversely with increasing range. A target, or scatterer, also has a farfield, in which the scattered field amplitude decreases inversely with increasing scattering distance. For a single-frequency component of a finite-duration sonar signal, the corresponding one-way amplitudes of the transmit signal and echo signal both decrease with range $r$ as $\exp (-\beta r) / r$, where $r$ is measured from the center of the co-located transmit and receive transducer, and where $\beta$ is the amplitude absorption coefficient in nepers per meter. In terms of the ordinary absorption coefficient $\alpha$ specified in decibels per meter, $\exp (-\beta r)=10^{(-\alpha r / 20)}$ and $\alpha=(20 \log \mathrm{e}) \beta \approx 8.686 \beta$.

This range dependence will also apply approximately to the entire sonar signal when $\beta$ is evaluated at the mean frequency. The approximation will be better for narrowband signals, worse for broadband signals.

In what follows, the fundamental difference between scattering by a single target and that by multiple targets is elaborated.

\section{Single scatterer}

If there is only one scatterer in the sampling volume, at range $r$ in the transmitter farfield, then the echo pressure amplitude varies with $r$ as $\exp (-2 \beta r) / r^{2}$, and the echo intensity varies as $\exp (-4 \beta r) / r^{4}$. This factor depends on $r$ but not on 
the properties of the scatterer. If the backscattering cross section $\sigma$ of the scatterer is to be measured, then the range dependence of the echo can be removed, or compensated, by multiplying the echo intensity by $r^{4} \exp (4 \beta r)$.

If the logarithmic measure of $\sigma$, namely the target strength, is of interest, then the range dependence of the echo strength is removed by adding the quantity $40 \log r$ $+2 \alpha r$. This gives rise to the name of the range compensation function used in target strength studies, namely "40 log $r$ $+2 \alpha r, " 6,13$ or "40 log $r$ " in parlance or in generic-sonar terminology according to Medwin and Clay. ${ }^{20}$

\section{Multiple scatterers}

If there are multiple scatterers or targets in the sampling volume, then the echo pressure amplitude is the simple sum of the individual amplitudes, in the neglect of extinction, which is a reasonable assumption except for strong scatterers at rather high numerical densities and extending over a considerable range interval. ${ }^{21}$ This sum will vary widely as a consequence of interference of overlapping echoes. When ensonified by a relatively long, pulsed sinusoidal signal, differences in scatterer range can be measured by phase, which is effectively randomly distributed. In the mean of a large number of observations, the total echo energy is the incoherent sum of the individual echo energies, without other effects, such as those of second-order scattering, which is usually negligible. ${ }^{22}$ Thus, the mean echo intensity will vary with the number of scatterers in the sampling volume and the mean backscattering cross section, i.e., as the mean volume backscattering coefficient. For a uniformly random distribution of scatterers, the number of scatterers in a conventional-sonar beam, hence with constant farfield beam pattern, will increase as $r^{2}$. Given the effects of geometric spreading and absorption, the echo intensity will vary as $\exp (-4 \beta r) / r^{2}$. This range dependence can be removed, or compensated, by multiplying the echo intensity by the factor $r^{2} \exp (4 \beta r)$.

In the logarithmic domain, the echo strength from a uniformly random distribution, or aggregation, of scatterers can be compensated for range by adding the quantity $20 \log r$ $+2 \alpha r$. This gives rise to the characteristic name of the range compensation function when the volume backscattering strength is to be measured. For generic sonars, according to Medwin and Clay, ${ }^{20}$ the additive quantity will be $20 \log r$.

\section{B. Range compensation for a parametric transmitter and conventional receiver}

A parametric sonar in the present context is an electromechanical system consisting of a parametric acoustic transmitter, a conventional receiver, and collocated primaryfrequency transmitting transducer and difference-frequency receiving transducer. The important general case of making measurements in the nearfield of the parametric transmitter and farfield of the conventional receiving transducer is thus considered. This mixed-field scenario introduces interesting complications in compensating for the range dependence of received echoes.

As explained in Sec. II A, the range dependence of received echoes by conventional sonar is relatively simple since measurements are typically made in the farfield of both the transmitting and receiving transducers. This is generally not the case with parametric sonars, where two additional effects must be considered: changing apparent source level and changing directional properties of the secondary, difference-frequency field generated by the nonlinear interaction of collinearly propagating primary fields, forming a so-called virtual endfire array. ${ }^{3}$ At the risk of redundancy, it is noted that backscattering is to be measured from scatterers located within the virtual endfire array itself, i.e., within its nearfield, where the differencefrequency field is being formed.

These effects can be quantified. With respect to transmission, the range dependence of the apparent source level of the secondary, difference-frequency field can be represented by the axial pressure $p_{T}(r)$. Its behavior differs qualitatively from that of the primary fields, whose nearfields are characterized by relatively rapid variations in axial pressure due to interference effects, as noted in Sec. I A. In contrast, the secondary, difference-frequency field will increase with increasing distance near the transducer, ultimately decreasing less rapidly with range than the primary fields do in their farfields. The directional properties of the secondary, difference-frequency field will also vary with range. This is represented through the range dependence of the transmit beam pattern $b_{T}(\mathbf{r})$, where $\mathbf{r}$ is the vector field position at range $r$ in the direction $\hat{r}$ as measured from the center of the co-located transmit and receive transducer.

As in Sec. II A, the fundamentally different cases of scattering by a single target and scattering by multiple targets are treated separately.

\section{Single scatterer}

If there is only one scatterer, or target, in the sampling volume, then the echo pressure amplitude will vary with $r$ as

$$
p_{T}(r) b_{T}^{1 / 2}(\mathbf{r}) \exp (-\beta r) / r
$$

The multiplicative factor to be applied to the echo intensity to remove range-dependent effects is thus the inverse square of this expression,

$$
R_{s}(\mathbf{r})=\left|p_{T}(r)\right|^{-2} b_{T}^{-1}(\mathbf{r}) r^{2} \exp (2 \beta r)
$$

For ordinary receivers, the target direction $\hat{r}$ will not be known, and the part of the range compensation function involving $b_{T}(\mathbf{r})$ must be neglected. For dual-beam, splitbeam, or other phase-measuring receivers, ${ }^{23}$ the target direction will be known and the full compensation can be made. Under conditions of full compensation, the compensated echo intensity will be proportional to the backscattering cross section of the single target.

In the farfield limit, $p_{T}(r)$ will vary as $\exp (-\beta r) / r$, and $b_{T}(\mathbf{r})$ will not depend on $r$. In this limiting case, full compensation can be achieved by applying the same formula described for conventional-sonar applications in Sec. II A 1. 


\section{Multiple scatterers}

If there are multiple scatterers, or targets, in the sampling volume, the echo pressure amplitude will vary with range $r$ as the sum of the individual echo pressure amplitudes given for the single-scatterer case in Sec. II B 1. For a uniformly random distribution of scatterers, with incoherent addition of individual scatterer echo energies as in Sec. II A 2, the number of scatterers will vary as $r^{2}$. The effective equivalent beam angle $\psi,{ }^{14,19}$ which measures the two-way integrated directionality, also varies with $\mathrm{r}$, as does the effective cross sectional area of the transmit and receive beams, $r^{2} \psi$. Combining the several effects, the received pressure amplitude will vary with $r$ as

$$
p_{T}(r) \psi^{1 / 2}(r) \exp (-\beta r)
$$

The range compensation function to be applied to the echo intensity is thus the inverse square, namely

$$
R_{m}(r)=\left|p_{T}(r)\right|^{-2} \psi^{-1}(r) \exp (2 \beta r)
$$

The result of applying this quantity to the echo intensity will be proportional to the volume backscattering strength of the target distribution.

In the farfield limit, $p_{T}(r)$ will vary as $\exp (-\beta r) / r$, and $\psi(r)$ will be a constant, independent of $r$. The function $R_{m}(r)$ will thus reduce to that for conventional-sonar farfield applications, with expression given in Sec. II A 2.

\section{Application of range compensation}

At one time, as noted in Sec. I A, range compensation would have been applied electrically or electronically through time-varied gain (TVG). Advances in digital signal processing now allow range compensation to be performed during postprocessing.

Digital-computer application of the function described in Eq. (2) for multiple scatterers is straightforward for narrowband difference frequencies. Its more general application for broadband difference frequencies is addressed in Sec. VI D, where applications of Eq. (1) for single scatterers are also addressed. In all cases, the domain of application is that of echo intensity or squared echo amplitude.

\section{CHARACTERISTICS OF A PARAMETRIC SONAR}

The nonlinearly generated difference-frequency field of a parametric sonar depends on the transmit frequencies, transmit power, and transducer array, hence on the characteristics and operating parameters of the particular sonar. These are described here for the parametric sonar used in the study described in Ref. 1, namely the Kongsberg Topographic Parametric Sonar (TOPAS) PS18 Parametric Sub-bottom Profiler. $^{2}$

This parametric sonar transmits primary frequencies in the band $15-21 \mathrm{kHz}$, generating secondary, or difference, frequencies in the overall band $0.5-6 \mathrm{kHz}$, but with most significant energy in the band $1-6 \mathrm{kHz}$. This energy is organized in continuous-wave $(\mathrm{CW})$ pulses, linear frequency-modulated (LFM) pulses, hyperbolic frequencymodulated (HFM) pulses, and Ricker pulses. The number of cycles in the difference-frequency $\mathrm{CW}$ pulses can be specified by the operator. Similarly, the frequency range and transmit pulse duration of the LFM and HFM pulses can be specified, as can the frequency and duration of the Ricker pulse.

The primary-frequency sonar transducer consists of a total of 256 identical, 60-mm-diameter circular elements arranged in a rectangular, nearly square array. There are 16 elements in each row, with center-center distance of $65 \mathrm{~mm}$. There are 16 rows, arranged in eight pairs. The center-center distance between corresponding elements in the two rows of a pair is $62.4 \mathrm{~mm}$. The center-center distance between adjacent paired rows is $142 \mathrm{~mm}$. The rows are oriented along the athwartship direction. The overall dimensions of the circumscribing rectangle are thus $1035 \mathrm{~mm}$ in the athwartship direction and $1116.4 \mathrm{~mm}$ in the alongship direction. The effective dimensions are reckoned as 1028.1 and $1109.5 \mathrm{~mm}$. Elements in adjacent rows of the same pair are connected in parallel, and the total number of individual channels is thus $8 \times 16$, allowing beam steering over an 80-deg sector. The overall array area is $11407 \mathrm{~cm}^{2}$. The net radiating area is $7238 \mathrm{~cm}^{2}$.

All of the elements are used in transmission with uniform excitation. The nearfield or Rayleigh distance $R_{0}$ is estimated as the ratio of the overall array area divided by the wavelength at the mean primary frequency $\nu_{0}=18 \mathrm{kHz}$, namely $R_{0}=13.9 \mathrm{~m}$.

The rms source level $\mathrm{SL}_{0}$ per single primary frequency is $237 \mathrm{~dB}$ re $1 \mu \mathrm{Pa} \cdot \mathrm{m}$. The scaled source level ${ }^{24}$ is $\mathrm{SL}_{0}+20 \log \nu_{0}$, where $\nu_{0}$ is the mean primary frequency in kilohertz. Since $\nu_{0}=18 \mathrm{kHz}$, the scaled source level is about $262 \mathrm{~dB}$ re $1 \mu \mathrm{Pa} \cdot \mathrm{kHz} \cdot \mathrm{m}$. Since the absorption coefficient $\alpha_{0}$ $\approx 0.00313 \mathrm{~dB} / \mathrm{m}$ at $\nu_{0}$, the absorption loss over the nearfield is $\alpha_{0} R_{0}=0.044 \mathrm{~dB}$. Thus the parametric source is absorptionlimited, with most of the difference-frequency generation occurring in the primary array farfield. ${ }^{24}$ The effective parametric array length $r_{\text {eff }}$ can be estimated in this absorptionlimited case from the graphical relationship given in Ref. 24, Fig. 7. The independent variable is $2 \beta_{0} R_{0} \nu_{0} / \nu$, where $\beta_{0}$ is the amplitude-absorption coefficient at $\nu_{0}$, namely $0.00036 \mathrm{~Np} / \mathrm{m}$, and $\mathrm{v}$ is the difference frequency. For $\nu$ in the range $1-6 \mathrm{kHz}$, values of $2 \beta_{0} R_{0} \nu_{0} / \nu$ are in the interval $0.18-0.03$, and the dependent variable in the cited figure, $2 \beta_{0} r_{\text {eff }}$ is about 0.7 , hence $r_{\text {eff }} \approx 1000 \mathrm{~m}$.

The transmit array directivity index ${ }^{19}$ at the $18-\mathrm{kHz}$ mean primary frequency has been computed according to the array geometry. It is about $33.48 \mathrm{~dB}$. The acoustic power density per primary frequency is thus $0.26 \mathrm{~W} / \mathrm{cm}^{2}$.

In reception, all array elements are used in the parametric sonar transducer installed on board R/V "G. O. Sars." The shape is effectively rectangular, with dimensions $1028.1 \mathrm{~mm}$ in the athwartship direction and $1109.5 \mathrm{~mm}$ in the alongship direction. Since the receiver is responding to echo signals in the difference-frequency band, the nearfield of the receiving array, of order $0.8 \mathrm{~m}$ at $1 \mathrm{kHz}$ and $4.6 \mathrm{~m}$ at $6 \mathrm{kHz}$, is much less than that of the transmitting array at the primary frequency, of order $14 \mathrm{~m}$. Thus the receiving array acts entirely conventionally at ranges of interest, which are $20 \mathrm{~m}$ or more. 


\section{MODELING THE PARAMETRIC SONAR NEARFIELD}

For determining the range compensation function of a parametric sonar as defined in Sec. II B as a combined parametric acoustic transmitter and conventional-sonar receiver, it is necessary to compute both the transmitted sound pressure level and the two-way equivalent beam angle over the difference-frequency band. These quantities can then be substituted in Eqs. (1) and (2) for the respective single- and multiple-scatterer functions.

\section{A. Algorithm and testing}

Essential to the several computations is a computational model for the nearfield of the parametric acoustic array generated by a finite aperture. Such a model has been developed by Moffett and Mellen, ${ }^{25}$ with primary documentation in Refs. 26 and 27, for an infinite homogeneous medium with constant sound speed, hence without refractive effects. It has been expressed in computer code, originally in FORTRAN. This has been updated in the interests of usability and to maintain currency with respect to changing computer platforms. The version used in the present computations is called CONVOL5. ${ }^{18}$

For validating the code in its present state, numerical computations were performed and compared, in Appendix A, against five cases for which both measurements and computations were reported. ${ }^{27-30}$ Those computations were performed using an earlier state or preliminary version of the code, with generally excellent agreement. Several of the cases treated arrays with properties not dissimilar to the sonar of particular interest here, described in Sec. III.

\section{B. Computational parameters}

In the following, computations with CONVOL5 have assumed that the transmitting array acts at the primary frequencies as a rectangular piston of dimensions 1028.1 $\times 1109.5 \mathrm{~mm}$. Computations of sound speed and acoustic absorption within CONVOL5 are based on the respective equations by Mackenzie ${ }^{31}$ and Francois and Garrison. ${ }^{32}$ The medium properties have been chosen consistently with the hydrography observed during the first applications of the parametric sonar to Norwegian spring-spawning herring (Clupea harengus) during cruises in December 2008 and November $2009,{ }^{1}$ including calibration. ${ }^{9}$ They are temperature $5^{\circ} \mathrm{C}$, salinity $35 \mathrm{ppt}$, and $\mathrm{pH} 8$. A mean depth of $200 \mathrm{~m}$ was chosen as representing the approximate mean depth of herring concentrations. The sound speed was thus $1473.9 \mathrm{~m} / \mathrm{s}$, which is considered representative of the total range of variation, $1471-1479 \mathrm{~m} / \mathrm{s}$.

\section{Nearfield characteristics}

Transmit beam patterns in the alongship plane are presented in Fig. 1 for the nonlinearly generated differencefrequency fields at 2 and $5 \mathrm{kHz}$. This is done for each of three ranges: 20,100, and $500 \mathrm{~m}$. These apply for the reported hydrographic conditions and depth of $200 \mathrm{~m}$. Beam patterns in the athwartship plane, not shown here, are slightly broader but otherwise rather similar.

The on-axis difference-frequency sound pressure levels at 2 and $5 \mathrm{kHz}$ are shown in Fig. 2(a) as functions of range over 20-1000 m. These sound pressure levels are absolute, assuming that the rms source level of a single primary frequency is $237 \mathrm{~dB}$ re $1 \mu \mathrm{Pa} \cdot \mathrm{m}$, as specified in Sec. III. The two-way equivalent beam angle at the same two frequencies is shown in Fig. 2(b), also as functions of range over 20-1000 m. This quantity is defined as the integral of the product of transmit and receiver beam patterns over all accessible angles, in this case over the 2-sr half-sphere on the exterior of the hull-mounted transducer array.

\section{RESULTS}

In evaluating or applying range compensation functions numerically, it is customary to normalize these to a reference range $r_{0}$. Thus the function for a single target given in Eq. (1) is normalized by the same function evaluated at $r_{0}$, namely $R_{s}\left(r_{0}\right)$. Similarly, the function for multiple targets given in Eq. (2) is divided by $R_{m}\left(r_{0}\right)$.

When range compensation functions were originally executed in time, by analog circuitry, it was common to assign $r_{0}$ to a minimum range, ${ }^{13}$ e.g., $1 \mathrm{~m}$. When later executed by
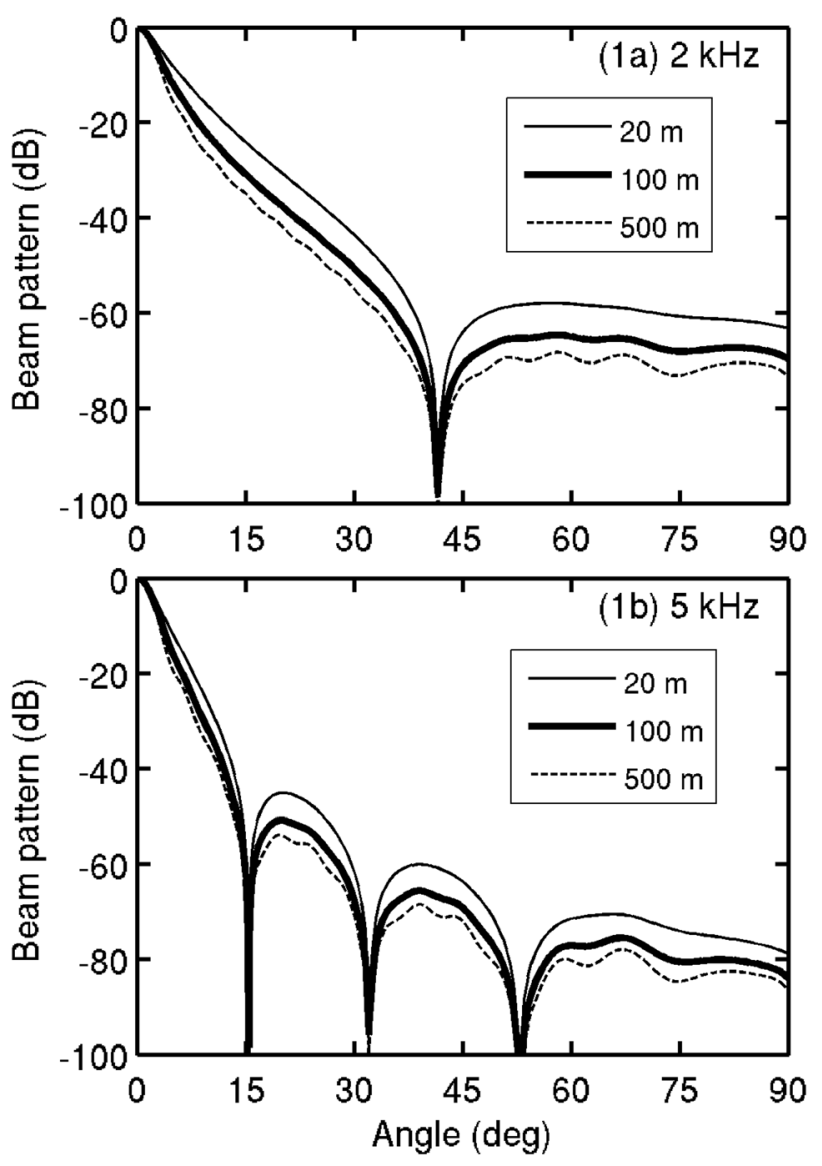

FIG. 1. Transmit beam patterns in the alongship plane of the TOPAS PS18 parametric sonar at the difference frequencies 2 and $5 \mathrm{kHz}$ for each of three ranges. 


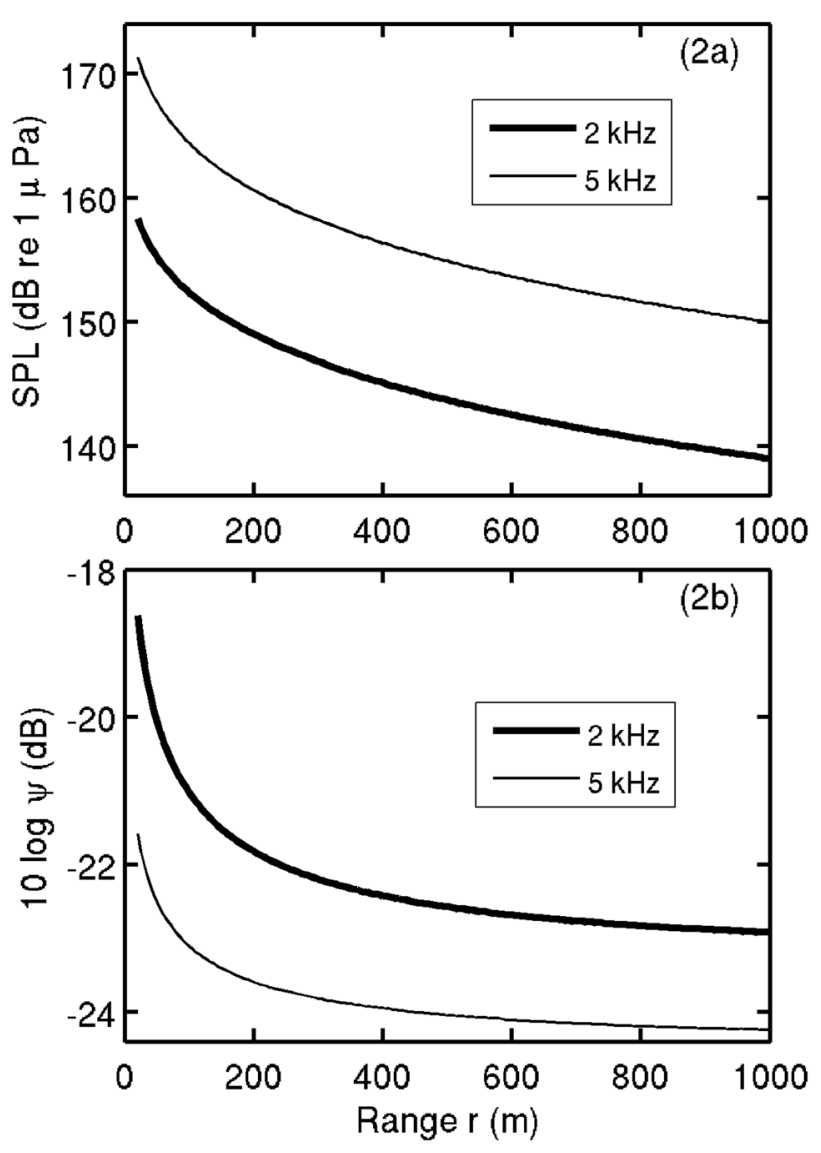

FIG. 2. On-axis transmit sound pressure level (SPL) and two-way equivalent beam angle $\psi$ of the TOPAS PS18 parametric sonar at the difference frequencies 2 and $5 \mathrm{kHz}$.

digital electronics, $r_{0}$ was assigned variously to a minimum range or to the maximum allowable range, ${ }^{20}$ e.g., 500 or $1000 \mathrm{~m}$. For present purposes, $r_{0}$ is chosen to be $20 \mathrm{~m}$, thus somewhat beyond the primary source Rayleigh distance of about $14 \mathrm{~m}$. The difference-frequency field is evaluated at 5 - $\mathrm{m}$ increments out to a maximum range of $1000 \mathrm{~m}$, which is also that of its effective length, estimated in Sec. III.

As already noted, the range compensation function for single targets requires knowledge of the target position, which is not generally known for simple receivers, but can be known for phase-measuring receivers. In evaluating the single-target range compensation function $R_{s}(\mathbf{r})$, it is assumed that $\mathbf{r}$ is known, hence $b_{T}(\mathbf{r})$ is known. Numerical results for the ratio

$$
\begin{aligned}
q_{s}(r) & \equiv R_{s}(r) / R_{s}\left(r_{0}\right) \\
& =\left|p_{T}\left(r_{0}\right) / p_{T}(r)\right|^{2}\left(r / r_{0}\right)^{2} \exp \left[2 \beta\left(r-r_{0}\right)\right],
\end{aligned}
$$

where $r_{0}=20 \mathrm{~m}$, are presented in Fig. 3 for the two difference frequencies, 2 and $5 \mathrm{kHz}$.

Evaluation of the multiple-target range compensation function, $R_{m}(r)$ given in Eq. (2), is less complicated, as knowledge of the target position is not required beyond that of the spatial distribution being uniformly random. This is no different from the situation with conventional sonars used to measure distributions of targets in the transducer farfield. Numerical results for the ratio

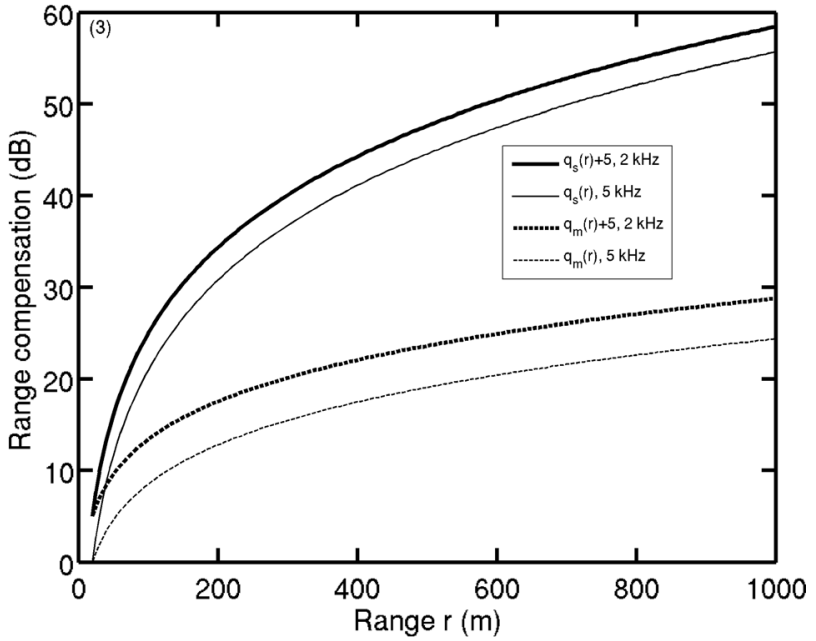

FIG. 3. Range compensation functions for single and multiple targets defined by the normalized quantities $q_{s}(r)$ and $q_{m}(r)$ given in Eqs. (3) and (4), respectively, for the TOPAS PS18 parametric sonar at the difference frequencies 2 and $5 \mathrm{kHz}$ for the assumed hydrographic state defined by temperature $5^{\circ} \mathrm{C}$, salinity $35 \mathrm{ppt}, \mathrm{pH} 8$, and depth $200 \mathrm{~m}$. To resolve the respective functions, those at $2 \mathrm{kHz}$ have been displaced by $5 \mathrm{~dB}$, as indicated in the legend.

$$
\begin{aligned}
q_{m}(r) & \equiv R_{m}(r) / R_{m}\left(r_{0}\right) \\
& =\left|p_{T}\left(r_{0}\right) / p_{T}(r)\right|^{2}\left[\psi\left(r_{0}\right) / \psi(r)\right] \exp \left[2 \beta\left(r-r_{0}\right)\right],
\end{aligned}
$$

where $r_{0}=20 \mathrm{~m}$, are included in Fig. 3 for both difference frequencies, 2 and $5 \mathrm{kHz}$.

The several computations underlying Fig. 3 have been repeated over the principal frequency band of interest, $1-6 \mathrm{kHz}$, in $1-\mathrm{kHz}$ increments. The respective ratios $q_{s}(r)$ and $q_{m}(r)$ are averaged in Fig. 4. Each of these averages is accompanied by the bounding functions, at 1 and $6 \mathrm{kHz}$.

\section{DISCUSSION}

\section{A. Comparing range compensation functions}

The particular results in Fig. 3 are interesting for showing the detailed nature of the range compensation functions to be applied to echo signals derived with the TOPAS PS18 parametric sonar described in Sec. III. These functions are not simple in the sense of range compensation functions applied to conventional-sonar signals with targets in the transducer farfield. The main reasons for this have already been elaborated: The difference-frequency source level and two-way equivalent beam angles are not constant as they would be with conventional-sonar farfield applications but range-dependent. In particular, the apparent source level of the difference-frequency transmit field increases with range $r$, which is evident in the on-axis sound pressure level computed in Fig. 2(a), which decreases less rapidly than $1 / r$. Also, the two-way equivalent beam angle $\psi$ decreases with increasing $r$, as indicated in Fig. 2(b), consistent with a sharpening of the difference-frequency beam with increasing range.

The several range compensation functions in Fig. 3 can be compared more directly with the respective functions 

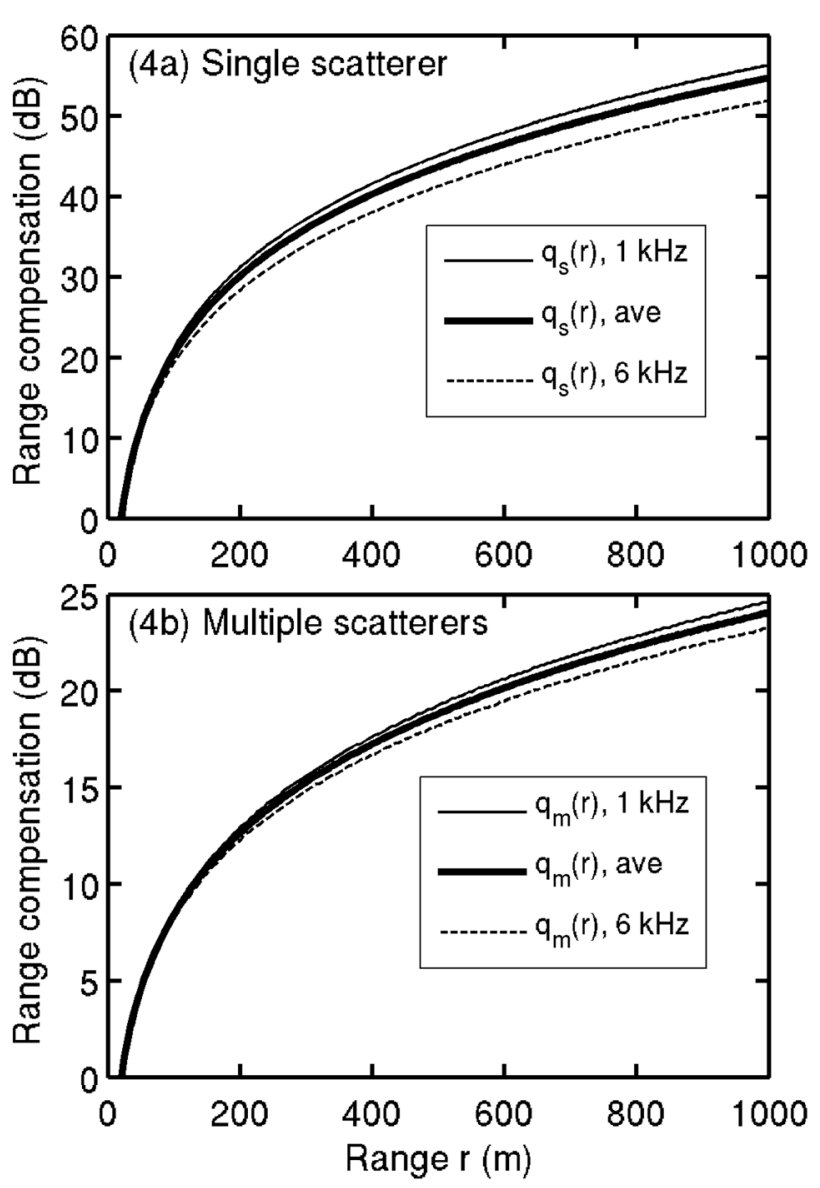

FIG. 4. Band-averaged and limiting range compensation functions for single and multiple targets defined by the normalized quantities $q_{s}(r)$ and $q_{m}(r)$ given in Eqs. (3) and (4), respectively, for the TOPAS PS18 parametric sonar. The averaging is performed over the difference-frequency band 1-6 kHz resolved at $1-\mathrm{kHz}$ intervals. The dependence of the range compensation function is monotonic with respect to frequency; the limiting functions at the difference frequencies 1 and $6 \mathrm{kHz}$ are shown. The assumed hydrographic state is defined by temperature $5^{\circ} \mathrm{C}$, salinity $35 \mathrm{ppt}, \mathrm{pH} 8$, and depth $200 \mathrm{~m}$.

associated with conventional-sonar measurements. If the functions in Eqs. (1) and (2) are represented by the power law $r^{\gamma}$, then $\gamma$ can be approximated by comparing the function at nearby ranges, say at $r \pm \Delta$, where $\Delta$ is a fraction of $r$. For the function $R_{m}(r)$, for example,

$$
\gamma_{m}(r) \approx \frac{\log \left[R_{m}(r+\Delta) / R_{m}(r-\Delta)\right]}{\log [(r+\Delta) /(r-\Delta)]}
$$

and similarly for $\gamma_{\mathrm{s}}$ associated with $R_{s}(r)$. This expression is very approximate, depending on the continuity of $R_{m}(r)$, thence on its constituent parts. The accuracy of CONVOL5 with respect to the on-axis pressure level is reckoned to be $\pm 0.5 \mathrm{~dB}$. This introduces a degree of jitter, or highfrequency variation, into $R_{m}(r)$ and ratio $q_{m}(r)$. To smooth this, $R_{m}(r+\Delta)$ was replaced in an ad hoc procedure by the arithmetic average of the three contiguous values $R_{m}(r+\Delta-5)$, $R_{m}(r+\Delta)$, and $R_{m}(r+\Delta+5)$, and similarly for $R_{m}(r-\Delta)$. The quantity $\Delta$ was equated to $25 \mathrm{~m}$. The computation was repeated for a total of 11 intervals: $[r-2 \Delta, r],[r-2 \Delta+5$, $r+5], \ldots,[r, r+2 \Delta]$, which were then averaged. At $2 \mathrm{kHz}$, $\gamma_{\mathrm{m}}=1.32$ at $100 \mathrm{~m}, 1.61$ at $500 \mathrm{~m}$, and 1.72 at $900 \mathrm{~m}$. At $5 \mathrm{kHz}, \gamma_{\mathrm{m}}=1.35,1.66$, and 1.80 at the respective ranges.

For a conventional sonar, $\gamma_{\mathrm{m}}$ would be somewhat greater than 2, increasing with $r$ because of the correction for absorption. Since $\alpha=0.000194 \mathrm{~dB} / \mathrm{m}$ at $2 \mathrm{kHz}$, the deviation of $\gamma_{\mathrm{m}}$ from exactly 2 at $1000 \mathrm{~m}$ is less than 0.024 . At $5 \mathrm{kHz}$, $\alpha=0.000364 \mathrm{~dB} / \mathrm{m}$ and the deviation of $\gamma_{\mathrm{m}}$ from 2 at $1000 \mathrm{~m}$ is less than 0.043 .

The several computations were repeated for the case of range compensation of a single scatterer at a known position in the transducer beam. At $2 \mathrm{kHz}, \gamma_{\mathrm{s}}=3.01$ at $100 \mathrm{~m}, 3.46$ at $500 \mathrm{~m}$, and 3.63 at $900 \mathrm{~m}$. At $5 \mathrm{kHz}, \gamma_{\mathrm{s}}=3.15,3.57$, and 3.74 at the respective ranges. For a conventional sonar, $\gamma_{\mathrm{s}}$ would be somewhat greater than 4 , with the deviation due to the correction for absorption. The maximum deviations of $\gamma_{\mathrm{s}}$ from 4 over the interval of interest are the same as for $\gamma_{\mathrm{m}}$, namely 0.024 and 0.043 at 2 and $5 \mathrm{kHz}$, respectively.

Another way of expressing the frequency dependence of the range compensation function is suggested by the presentation of the functions in Fig. 4. The central functions are the averages of $q_{m}(r)$ and $q_{s}(r)$ with respect to frequency over the band $1-6 \mathrm{kHz}$. They are accompanied by the respective minimum- and maximum-valued functions. For the normalized range compensation function applying to multiple scatterers, $q_{m}(r)$ in Eq. (4), the total variation is $8.24-8.59 \mathrm{~dB}$ at $100 \mathrm{~m}, 18.24-19.28 \mathrm{~dB}$ at $500 \mathrm{~m}, 22.46-23.82 \mathrm{~dB}$ at $900 \mathrm{~m}$, and $23.21-24.58 \mathrm{~dB}$ at $1000 \mathrm{~m}$. For the normalized range compensation function applying to single scatterers at known position in the transducer beam, $q_{s}(r)$ in Eq. (3), the total variation is $19.29-21.23 \mathrm{~dB}$ at $100 \mathrm{~m}, 41.29-45.10 \mathrm{~dB}$ at $500 \mathrm{~m}$, $50.22-54.59 \mathrm{~dB}$ at $900 \mathrm{~m}$, and $51.84-56.26 \mathrm{~dB}$ at $1000 \mathrm{~m}$.

\section{B. Computational accuracy}

A number of approximations have been used in computing the range compensation functions underlying Figs. 3 and 4, which must affect their quality. This cannot be gauged exactly, owing to the complicated nature of the differencefrequency field in the nearfield of the virtual endfire array. This difference-frequency field has been computed using the established code CONVOL5, ${ }^{18}$ with already noted error in computations of the on-axis pressure level of $\pm 0.5 \mathrm{~dB}$. Further computational error can be expected from the assumption of an overall rectangular shape for the transducer, whereas the actual transducer is a rather dense rectangular array of circular pistons. The effect of the so-called aperture factor $^{28,33,34}$ is examined in Appendix B. The principal finding is that use of the rectangular-piston approximation incurs errors of order several tenths of a decibel in reception. It is believed that this also applies in transmission.

\section{Dependences of range compensation functions}

Range compensation functions for parametric sonar, unlike those for conventional sonars when operated in the transducer farfield, depend on the particular source geometry and operating conditions. Generalizing the range compensation functions for the arbitrary parametric sonar, as has been done for certain performance measures, ${ }^{24}$ would be a considerable undertaking of questionable value given the rather 
small number of parametric sonars that measure watercolumn backscattering. In lieu of such a generalization, the sensitivity of range compensation functions to four factors beyond those of frequency, described in Figs. 3 and 4 and Sec. VI A, is examined. This is done at a single frequency, $3 \mathrm{kHz}$, as representing an intermediate frequency in the difference-frequency band of the TOPAS PS18 parametric sonar. The basic question being addressed is how similar, or different, are the functions when a significant characteristic is changed. Except for the characteristic being varied, the original operating parameters of the particular parametric sonar were maintained, with exceptions noted.

\section{Transducer shape}

For convenience, the dependence on transducer shape was investigated first. The range compensation functions were determined for an equivalent-area circular piston transducer, relative to the overall radiating area of the TOPAS PS18 transducer, hence with diameter $1205.1 \mathrm{~mm}$. The parametric transmit beam pattern at $3 \mathrm{kHz}$ of the circular piston was modeled with CONVOL5, otherwise using the same operating parameters as described in Sec. III. The respective beam patterns differed as expected: the main lobe of the circular piston was intermediate to those of the rectangular TOPAS PS18 source transducer aligned with the sides, while the sidelobes of the circular piston were lower than those of the rectangular source. In terms of the transmit directivity index, this was 33.29 and $33.48 \mathrm{~dB}$ at $100 \mathrm{~m}$ for the respective circular and rectangular sources. The respective values of the receive directivity index were 17.84 and $17.86 \mathrm{~dB}$ at all ranges of interest. Values of $\psi$ agreed to within $0.03 \mathrm{~dB}$ for ranges out to $500 \mathrm{~m}$ and to within $0.05 \mathrm{~dB}$ for ranges out to $1000 \mathrm{~m}$. Values of $q_{m}(r)$ in Eq. (4) agreed to within about $0.1 \mathrm{~dB}$ at ranges to $500 \mathrm{~m}$ and $0.3 \mathrm{~dB}$ at ranges to $1000 \mathrm{~m}$, with those of the circular piston being consistently higher. Values of $q_{s}(r)$ in Eq. (3) agreed to within about $0.1 \mathrm{~dB}$ at ranges to $500 \mathrm{~m}$ and $0.4 \mathrm{~dB}$ at ranges to $1000 \mathrm{~m}$, again with those of the circular piston being consistently higher.

\section{Hydrography}

Given the similarity of the range compensation functions for the two transducer shapes, that of the circular piston was used to investigate the dependence on temperature, hence medium sound speed. As noted in Sec. IV A, CONVOL5 is limited to an infinite homogeneous medium with constant sound speed. Thus the investigations of the effect of hydrography were undertaken through a series of computations, with each set performed for a constant value of medium sound speed.

The baseline computations were performed for a temperature of $5^{\circ} \mathrm{C}$, with salinity $35 \mathrm{ppt}$ and $\mathrm{pH}=8$. At $0^{\circ} \mathrm{C}$, $q_{m}(r)$ differed from that at $5^{\circ} \mathrm{C}$ by $0.1 \mathrm{~dB}$ at $100 \mathrm{~m}$ and $0.2 \mathrm{~dB}$ at $500 \mathrm{~m}$, and similarly for $q_{s}(r)$. At $10^{\circ} \mathrm{C}$, the two functions differed from their respective counterparts at $5^{\circ} \mathrm{C}$ by less than $0.2 \mathrm{~dB}$ at all ranges. At $20^{\circ} \mathrm{C}$, the functions differed from their respective counterparts at $5^{\circ} \mathrm{C}$ by less than $0.6 \mathrm{~dB}$ at all ranges to $1000 \mathrm{~m}$. Thus, in the neglect of temperature over the range $0-20^{\circ} \mathrm{C}$, a maximum error of less than $0.6 \mathrm{~dB}$ is incurred by the use of range compensation functions computed for $5^{\circ} \mathrm{C}$. The trend in $q_{m}(r)$ and $q_{s}(r)$ with respect to temperature is monotonically increasing with $r$. The dependence of the range compensation functions on the sound speed in an infinite homogeneous medium may be regarded as relatively weak.

Effects of small-scale random variations in sound speed, or refractive index, might be expected to be still weaker, but the nature of the inhomogeneities must be considered more closely. If the concentration and spatial extent of inhomogeneities are large, or if high contrast in sound speed and/or mass density is involved, as would be the case with gas bubbles, then substantial effects might be expected due to an increase in the nonlinearity of the medium and radically changed dispersion. ${ }^{35,36}$ The nonlinear character of bubble oscillations in the presence of multiple sound fields, e.g., two primary waves, will also generate difference-frequency waves at single bubbles, but such effects are expected to be significantly less. ${ }^{37}$ Turbulence may also be a source of perceptible nonlinear effects, as in the example of nonlinear scattering of crossed beams, ${ }^{38,39}$ further recognizing the particular influence of air bubbles. ${ }^{40}$

\section{Acoustic power density}

Based on the exact TOPAS PS18 transducer geometry as an array of 256 identical, 60-mm-diameter, circular pistons and rms source level per primary frequency of $237 \mathrm{~dB}$ re $1 \mu \mathrm{Pa} \cdot \mathrm{m}$, the acoustic power density is $0.26 \mathrm{~W} / \mathrm{cm}^{2}$ per frequency. For a circular piston transducer of diameter $1205.1 \mathrm{~mm}$, the rms source level per frequency with this same power density would be $238.82 \mathrm{~dB}$ re $1 \mu \mathrm{Pa} \cdot \mathrm{m}$. The maximum difference in $q_{m}(r)$ for the two source levels is less than $0.3 \mathrm{~dB}$ at all ranges to $1000 \mathrm{~m}$; that in $q_{s}(r)$ is less than $0.4 \mathrm{~dB}$ for the same ranges.

For the acoustic power density $0.1 \mathrm{~W} / \mathrm{cm}^{2}$, the $\mathrm{rms}$ source level per primary frequency is $234.67 \mathrm{~dB}$ re $1 \mu \mathrm{Pa} \cdot \mathrm{m}$, and the maximum difference in range compensation functions relative to the reference power density of $0.26 \mathrm{~W} / \mathrm{cm}^{2}$ is less than $0.5 \mathrm{~dB}$ for $q_{m}(r)$ and $0.6 \mathrm{~dB}$ for $q_{s}(r)$ at all ranges to $1000 \mathrm{~m}$. For the acoustic power density $1 \mathrm{~W} / \mathrm{cm}^{2}$, the $\mathrm{rms}$ source level per primary frequency is $244.67 \mathrm{~dB}$ re $1 \mu \mathrm{Pa} \cdot \mathrm{m}$. This represents a practical upper limit to the power density with $\nu_{0}=18 \mathrm{kHz},{ }^{41}$ given that the parametric sonar is typically excited simultaneously by two primary frequencies. The respective difference in the two functions relative to the reference power density is less than $1.7 \mathrm{~dB}$ for $q_{m}(r)$ and $2.2 \mathrm{~dB}$ for $q_{s}(r)$ at all ranges to $1000 \mathrm{~m}$.

\section{Transducer size}

It is assumed that the acoustic power density is identical to that of specified TOPAS PS18 operation, hence $0.26 \mathrm{~W} / \mathrm{cm}^{2}$. For a 381.1-mm-diameter circular piston, hence with area that is $10 \%$ of that of the 1205.1 -mm-diameter reference circular piston assumed in this section, $q_{m}(r)$ is increased by $1.3 \mathrm{~dB}$ at $100 \mathrm{~m}, 2.0 \mathrm{~dB}$ at $500 \mathrm{~m}$, and $2.2 \mathrm{~dB}$ at $1000 \mathrm{~m}$ relative to the larger reference transducer. Similarly, $q_{s}(r)$ increases by $2.5,4.0$, and $4.3 \mathrm{~dB}$ at the same respective ranges. In general, the relative range compensation increases with decreasing transducer size. 


\section{Application of computed functions}

The results presented in Figs. 3 and 4, as well as similar ones for other single frequencies in the differencefrequency band, are suitable for direct application to socalled continuous-wave $(\mathrm{CW})$ signals generated by the parametric sonar of interest, at least for the assumed hydrographic conditions. For application to other, broadband signals, such as linear or hyperbolic frequency-modulated (FM) signals or Ricker pulses, the individual range compensation functions would require consistent, integrated application over the frequency band. This would also require allowance for differences in transmitter and receiver sensitivities with frequency.

For applications of range compensation at other temperatures and/or acoustic power densities, it may be necessary or advisable to recompute the function. Whether this is necessary may be gauged from the estimated sensitivities in Sec. VI C.

The actual application of range compensation to echoes from single scatterers deserves attention. If range compensation were to be applied over the finite duration of the echo signal in the manner of time-varied gain, this would introduce a bias into the echo processing and distort the characterization and comparison of target echoes. If the range to a single target were identified by matchedfiltering, or more precisely cross correlation of the echo signal with the effective difference-frequency transmit signal, then the numerical value of the appropriate range compensation function, namely $q_{s}(r)$ in Eq. (3), could be applied as a simple multiplying factor to the entire signal or to its energy. The potential bias, if not avoided, would be greatest for targets ensonified at short ranges by longduration transmit signals.

As mentioned in Sec. II C, the application of range compensation functions as described in Eqs. (1) and (2), or Eqs. (3) and (4), is rendered in the echo-intensity domain. If a calibration has been performed for a narrowband difference frequency at a fixed range, then the parametric sonar can be used to measure the echo strength of an unknown target or the volume backscattering strength of a number of randomly distributed targets for the same frequency and range as during the calibration. The derived calibration constant or factor can be used to scale the respective range compensation function. With digital-computer application of range compensation, measurements of echo intensity are rendered absolute at all ranges.

\section{E. Future work}

The TOPAS PS18 parametric sonar is already being used to measure one of the stocks of Atlantic herring (Clupea harengus), namely the spawning stock of Norwegian spring-spawning herring. ${ }^{1}$ This has been done during cruises in the wintering area near 71 N, $15 \mathrm{E}$ in both December 2008 and November 2009. The numerical results for the ratio $q_{m}(r) \equiv R_{m}(r) / R_{m}\left(r_{0}\right)$ in Eq. (4) will be applied to echo data on herring aggregations and schools observed in situ during these cruises. This will remove first-order range dependent effects, enabling echograms to be expressed in relative units of volume backscattering. This will enable spectra to be derived that may reveal swimbladder resonances, ${ }^{4,5}$ including depth and depth-history effects.

When available, results for the standard-target calibration $^{42,43}$ performed during the first cruise ${ }^{9}$ will be used in frequency-dependent scaling factors so that the rangecompensated echograms can be expressed in absolute physical units of volume backscattering strength. This will enable measurements made in the mid-frequency sonar band to be compared with echo measurements made at ordinary ultrasonic frequencies, e.g., 18, 38, 120, and $200 \mathrm{kHz}$, among others. Because of differences in beamwidth, it may be possible to compare echo data derived with the very directional difference-frequency parametric sonar beam and echo data derived with the less directional beams of conventional scientific echo sounders. This comparison is expected to enable possible behavioral effects ${ }^{44,45}$ to be quantified, and midfrequency target strengths of herring to be inferred, allowing application of the echo integration method ${ }^{6,7}$ at kilohertz frequencies.

\section{ACKNOWLEDGMENTS}

M. B. Moffett has graciously provided the CONVOL5 code and answered diverse inquiries on this. J. Dybedal is thanked for essential information on the TOPAS PS18 parametric sonar. Y.-T. Lin is thanked for assistance with equation-editing, A. Newhall for guidance in figure preparation, and M. Parmenter for assistance with text-editing. This work was supported by ONR Award No. N000140910482.

\section{APPENDIX A. COMPARISON OF CONVOL5 MODEL COMPUTATIONS WITH HISTORICAL MEASUREMENTS AND COMPUTATIONS}

\section{Case 1. Beam pattern of circular source per Moffett and Mellen (1976) ${ }^{28}$}

The parametric projector consisted of a 102-mm-diameter circular piston operating at $245 \pm 25 \mathrm{kHz}$, with rms source level $\mathrm{SL}_{0}=217.5 \mathrm{~dB}$ re $1 \mu \mathrm{Pa} \cdot \mathrm{m}$ for each primary frequency. The immersion medium was sea water, in the Millstone Quarry, Waterford, CT, with salinity $29.5 \mathrm{ppt}$ and temperature $30^{\circ} \mathrm{C}$. The parametric transmit beam pattern at the difference frequency $50 \mathrm{kHz}$ was measured at $85-\mathrm{m}$ range. ${ }^{28}$ Agreement of model computations using CONVOL5 with the results presented in Ref. 28, Fig. 2, was excellent.

The scaled source level is $\mathrm{SL}_{0}+20 \log \nu_{0}$, where $\nu_{0}$ is the mean primary frequency in kilohertz, i.e., $265.3 \mathrm{~dB}$ re $1 \mu \mathrm{Pa} \cdot \mathrm{kHz} \cdot \mathrm{m}$. The nearfield or Rayleigh distance $R_{0}$ is estimated as the transducer area divided by the wavelength at the mean primary frequency $\nu_{0}=245 \mathrm{kHz}$, namely $1.3 \mathrm{~m}$. The product of the absorption coefficient $\alpha_{0}=0.096 \mathrm{~dB} / \mathrm{m}$ at $\nu_{0}$, and $R_{0}$ is thus $0.12 \mathrm{~dB}$. The particular parametric transmitter is absorption-limited with most of the difference-frequency sound being generated in the farfield of the primary source. ${ }^{28}$ As noted in Sect. III, the effective length of an absorptionlimited parametric array can be estimated from the graphical 
relationship given in Ref. 24, Fig. 7. The independent variable is $2 \beta_{0} R_{0} \nu_{0} / \nu$, where $\beta_{0}$ is the amplitude-absorption coefficient $\alpha_{0} / 8.686=0.011 \mathrm{~Np} / \mathrm{m}$ and $\nu$ is the difference frequency. For $\nu=50 \mathrm{kHz}, 2 \beta_{0} R_{0} \nu_{0} / \nu=0.14$ and $2 \beta_{0} r_{\text {eff }}=0.7$, hence $r_{\text {eff }}=32 \mathrm{~m}$.

For the specified transducer dimensions, the transmit directivity index at $\nu_{0}$ is $34.15 \mathrm{~dB}$. The acoustic power density per primary frequency is thus $0.21 \mathrm{~W} / \mathrm{cm}^{2}$.

\section{Case 2. Beam patterns of circular source per Moffett and Mellen (1977) ${ }^{29}$}

The parametric projector was a 254-mm-diameter circular piston operating at the mean primary frequency $245 \mathrm{kHz}$ with each of two rms primary source levels, 220 and $236 \mathrm{~dB}$ re $1 \mu \mathrm{Pa} \cdot \mathrm{m}$ for each primary frequency. The sea water immersion medium and measurement range of $84.5 \mathrm{~m}$ were essentially the same as in Case 1 . Numerical results obtained with CONVOL5 at each of three difference frequencies, 50, 25 , and $12.5 \mathrm{kHz}$, agreed with the respective measurements and model computations presented in Ref. 29, Figs. 10(a) and 10(b), for the two source levels. Modeling results for the $5-\mathrm{kHz}$ difference frequency agreed, noting differences with the original measurements.

The scaled source levels are 268 and $284 \mathrm{~dB}$ re $1 \mu \mathrm{Pa} \cdot \mathrm{kHz} \cdot \mathrm{m}$. The first describes an absorption-limited array; the second, a saturation-limited array where there is substantial harmonic generation in the primary-array nearfield. The Rayleigh distance at $\nu_{0}=245 \mathrm{kHz}$ is $8.1 \mathrm{~m}$, and $\alpha_{0} R_{0}=0.77 \mathrm{~dB}$. For the lesser of the two source levels, most of the difference-frequency generation occurs in the primary array farfield. Thus $r_{\text {eff }}=0.6 /\left(2 \beta_{0}\right)=27 \mathrm{~m}$ at $\nu=50 \mathrm{kHz}$ and $r_{\text {eff }}=9 /\left(2 \beta_{0}\right)=410 \mathrm{~m}$ at $\nu=5 \mathrm{kHz}$. Measurements repeated by Moffett and Mellen at about 30-m range were reported as being very similar to those at $84.5-\mathrm{m}$ range. At $50 \mathrm{kHz}$, at least, these observations are consistent with the estimated effective array length.

The transmit directivity index at $\nu_{0}=245 \mathrm{kHz}$ is $42.19 \mathrm{~dB}$. The acoustic power density per primary frequency is thus 0.01 and $0.40 \mathrm{~W} / \mathrm{cm}^{2}$ for the respective source levels.

\section{Case 3. Beamwidths of two rectangular sources per Moffett et al. (1978) ${ }^{30}$}

The dimensions of the respective parametric source apertures were $102 \times 81 \mathrm{~mm}$ and $205 \times 39 \mathrm{~mm}$. The mean primary frequency was $410 \mathrm{kHz}$. Measurements were made of the source level and beamwidths in fresh water, in Dodge Pond, at temperature $3.1^{\circ} \mathrm{C}$. $^{30}$ The effective rms primary source level per primary frequency was in the approximate range $200-230 \mathrm{~dB}$ re $1 \mu \mathrm{Pa} \cdot \mathrm{m}$. Beamwidths computed with CONVOL5 agreed with those measured at $78 \mathrm{~m}$ to within limits of readability as presented in Ref. 30, Fig. 7.

\section{Case 4. Beam patterns of rectangular sources per Moffett and Mellen (1981) ${ }^{27}$}

The dimensions of the parametric sources were $530 \times 440 \mathrm{~mm}$. The rms primary frequency was $24 \mathrm{kHz}$ and rms source level, $228 \mathrm{~dB}$ re $1 \mu \mathrm{Pa} \cdot \mathrm{m}$ at each primary frequency. Measurements of the difference-frequency field at $1 \mathrm{kHz}$ were made at $43.5-\mathrm{m}$ range in fresh water, in Seneca Lake, temperature $4{ }^{\circ} \mathrm{C} .{ }^{27}$ Agreement of model computations from CONVOL5 with results presented in Ref. 27, Fig. 6, were excellent.

The scaled source level is about $256 \mathrm{~dB}$ re $1 \mu \mathrm{Pa} \cdot \mathrm{kHz} \cdot \mathrm{m}$. The Rayleigh distance at $\nu_{0}=24 \mathrm{kHz}$ is $3.9 \mathrm{~m}$, and $\alpha_{0} R_{0}$ $=0.00091 \mathrm{~dB}$. The parametric source is thus absorptionlimited with most of the difference-frequency generation occurring in the primary source farfield. The parameter determining the effective array length is $2 \beta_{0} R_{0} \nu_{0} / \nu=0.005$ for $\nu=1 \mathrm{kHz}$. Thus according to the cited figure, $2 \beta_{0} r_{\mathrm{eff}}=0.55$, hence $r_{\text {eff }}=110 \mathrm{~m}$. The transmit directivity index of the rectangular source at $\nu_{0}$ is $29.70 \mathrm{~dB}$. The acoustic power density is thus $0.26 \mathrm{~W} / \mathrm{cm}^{2}$.

\section{Case 5. Beam patterns of circular source per Moffett and Mellen (1981) ${ }^{27}$}

The diameter of the source was $950 \mathrm{~mm}$. The mean primary frequency was $64 \mathrm{kHz}$, and the rms source level $\mathrm{SL}_{0}$ was $245 \mathrm{~dB}$ re $1 \mu \mathrm{Pa} \cdot \mathrm{m}$ at each primary frequency. Measurements of the difference-frequency field at $3.5 \mathrm{kHz}$ were made at $82-\mathrm{m}$ range in a sea water quarry with assumed salinity $29.5 \mathrm{ppt}$ and assumed temperature $4{ }^{\circ} \mathrm{C} .{ }^{27}$ Agreement of model computations with CONVOL5 with results presented in Ref. 27, Fig. 7, was excellent.

The scaled source level was $281 \mathrm{~dB}$ re $1 \mu \mathrm{Pa} \cdot \mathrm{kHz} \cdot \mathrm{m}$. The Rayleigh distance at $\nu_{0}=64 \mathrm{kHz}$ is $31.9 \mathrm{~m}$ and $\alpha_{0} R_{0}=0.52 \mathrm{~dB}$. The parametric array is thus saturationlimited with substantial difference-frequency generation in the nearfield of the primary source. The transmit directivity index is $42.57 \mathrm{~dB}$, and the acoustic power density is $0.24 \mathrm{~W} / \mathrm{cm}^{2}$.

\section{APPENDIX B. APERTURE FACTOR}

The difference-frequency field generated by a parametric acoustic array is modulated by the transducer, or aperture, that launches the primary-frequency field..$^{28,33,34}$ Because of the present need to compute the nearfield of the parametric sonar, which is appreciated to involve three-dimensional integration of an oscillating function, ${ }^{26}$ the TOPAS PS18 transducer is represented as a simple rectangular piston. How good is this approximation?

The answer cannot be given definitively, but may be suggested by examining the so-called aperture factor, or beam pattern associated with the parametric sonar transducer array acting conventionally at the difference frequency. For convenience, the array is assumed to lie in the $(x, y)$ plane, with $x$ axis aligned in the athwartship direction and $y$ axis in the alongship direction of a rectangular coordinate system with $z$ axis normal to the transducer plane. The field direction $\hat{k}$ is specified by two angles. The polar angle is $\theta=\cos ^{-1}$ $(\hat{k} \cdot \hat{z})$. For the unit vector $\hat{u}=[\mathbf{k}-(\mathbf{k} \cdot \hat{z}) \hat{z}] /|\mathbf{k}-(\mathbf{k} \cdot \hat{z}) \hat{z}|$, where $\mathbf{k}$ is the wavevector, the azimuthal angle $\phi=\cos ^{-1}$ $(\hat{u} \cdot \hat{x})$. For dimensional computations, the wavenumber $k=2 \pi / \lambda$, where $\lambda$ is the acoustic wavelength.

The beam pattern of an ideal rectangular piston of side lengths $l_{1}$ and $l_{2}$ in the respective $x$ and $y$ directions is 


$$
b=\left[\frac{\sin \left(\frac{1}{2} k l_{1} \sin \theta \cos \phi\right)}{\frac{1}{2} k l_{1} \sin \theta \cos \phi} \frac{\sin \left(\frac{1}{2} k l_{2} \sin \theta \sin \phi\right)}{\frac{1}{2} k l_{2} \sin \theta \sin \phi}\right]^{2}
$$

For the TOPAS transducer array, $l_{l}=1028.1 \mathrm{~mm}$ and $l_{2}=1109.5 \mathrm{~mm}$.

This is to be contrasted with the beam pattern of the rectangular array of identical circular elements described in Sect. III, namely the triple product $b_{1} b_{x} b_{y}$, where $b_{1}$ is the beam pattern of a single circular piston of radius $a=30 \mathrm{~mm}$, where $J_{1}(x)$ is a Bessel function of order 1:

$$
b_{1}=\left[\frac{2 J_{1}(k a \sin \theta)}{k a \sin \theta}\right]^{2}
$$

where $a=30 \mathrm{~mm}$ is the piston radius. The second factor describes the beam pattern of a line array of point elements in the athwartship direction,

$$
b_{x}=\left[\frac{\sin \left(\frac{n}{2} k \xi \sin \theta \cos \phi\right)}{\frac{n}{2} k \xi \sin \theta \cos \phi}\right]^{2}
$$

where $n=16$ is the number of elements evenly spaced at center-center distance $\xi=65 \mathrm{~mm}$. The third factor describes the beam pattern of a linear array of pairs of point elements in the alongship direction,

$$
b_{y}=\left[\frac{\sin \left(\frac{m_{1}}{2} k \eta_{1} \sin \theta \sin \phi\right)}{\frac{m_{1}}{2} k \eta_{1} \sin \theta \sin \phi} \frac{\sin \left(\frac{m_{2}}{2} k \eta_{2} \sin \theta \sin \phi\right)}{\frac{m_{2}}{2} k \eta_{2} \sin \theta \sin \phi}\right]^{2},
$$

where $m_{1}=2$ for the pair with center-center distance $\eta_{1}=62.4 \mathrm{~mm}$, and $\mathrm{m}_{2}=8$ for the number of pairs with pair center-pair center distance $\eta_{2}=142 \mathrm{~mm}$.

The beam patterns of the rectangular piston and the array of elements have both been computed and compared through the directivity index DI for the assumed sound speed $1473.9 \mathrm{~m} / \mathrm{s}$. The respective values of DI at $2 \mathrm{kHz}$ are 14.23 and $14.45 \mathrm{~dB}$, and at $5 \mathrm{kHz}, 22.14$ and $22.26 \mathrm{~dB}$. That is, these values are relatively comparable, providing some justification for approximation of the particular parametric sonar transducer array by a rectangular piston.

${ }^{1}$ O. R. Godø, K. G. Foote, J. Dybedal, E. Tenningen, and R. Patel, "Detecting Atlantic herring by parametric sonar," J. Acoust. Soc. Am. 127, EL153-EL159 (2010).

${ }^{2}$ J. Dybedal, "TOPAS: parametric end-fire array used in offshore applications," in Advances in Nonlinear Acoustics, edited by H. Hobæk (World Scientific, Singapore, 1993), pp. 264-275.

${ }^{3}$ P. J. Westervelt, "Parametric acoustic array," J. Acoust. Soc. Am. 35, 535-537 (1963).

${ }^{4}$ D. V. Holliday, "Resonance structure in echoes from schooled pelagic fish," J. Acoust. Soc. Am. 51, 1322-1332 (1972).

${ }^{5}$ A. Løvik and J. M. Hovem, "Experimental investigation of swimbladder resonance in fishes," J. Acoust. Soc. Am. 66, 850-854 (1979).
${ }^{6} \mathrm{~S}$. T. Forbes and O. Nakken, eds., "Manual of methods for fisheries resource survey and appraisal. Part 2. The use of acoustic instruments for fish detection and abundance estimation," FAO Man. Fish. Sci. 5, 138 pp. (1972).

${ }^{7}$ K. G. Foote and T. K. Stanton, "Acoustical methods," in ICES Zooplankton Methodology Manual, edited by R. Harris, P. Wiebe, J. Lenz, H. R. Skjoldal, and M. Huntley (Academic, San Diego, 2000), Chap. 6, pp. 223-258.

${ }^{8}$ O. R. Godø, R. J. Korneliussen, and K. G. Foote, "Quantifying fish behavior with high-frequency scientific echo sounder and parametric sonar (A)," J. Acoust. Soc. Am. 127, 1729 (2010).

${ }^{9}$ K. G. Foote, J. Dybedal, and E. Tenningen, "Standard-target calibration of a parametric sonar over the difference-frequency band, 1-6 kilohertz (A)," J. Acoust. Soc. Am. 125, 2718 (2009).

${ }^{10}$ K. G. Foote, "Range compensation function for echo integration in transducer near fields, with special reference to parametric sonar (A)," J. Acoust. Soc. Am. 125, 2718 (2009).

${ }^{11}$ C. H. Sherman and J. L. Butler, Transducers and Arrays for Underwater Sound (Springer, New York, 2007), pp. 456, 530, 531.

${ }^{12}$ A. J. Burridge, P. G. Griffiths, J. W. R. Griffiths, and D. W. Hoare, "Timevaried gain echo-sounder receiver systems for use with fish echo signal processing equipment," Rapp. P.-V. Réun.-Cons. Int. Explor. Mer 170, 83-87 (1977).

${ }^{13}$ R. B. Mitson, Fisheries Sonar (Incorporating Underwater Observation Using Sonar by D G Tucker) (Fishing News Books, Farnham, Surrey, 1983), pp. 127-129, 246, 247.

${ }^{14}$ D. N. MacLennan, "Acoustical measurement of fish abundance," J. Acoust. Soc. Am. 87, 1-15 (1990).

${ }^{15}$ K. G. Foote, H. P. Knudsen, R. J. Korneliussen, P. E. Nordb $\varnothing$, and K. Røang, "Postprocessing system for echo sounder data," J. Acoust. Soc. Am. 90, 37-47 (1991).

${ }^{16}$ L. N. Andersen, "The new Simrad EK60 scientific echo sounder system (A)," J. Acoust. Soc. Am. 109, 2336 (2001).

${ }^{17}$ T. G. Muir and J. G. Willette, "Parametric acoustic transmitting arrays," J. Acoust. Soc. Am. 52, 1481-1486 (1972).

${ }^{18}$ M. B. Moffett and H. C. Robinson, "User's manual for the CONVOL5 computer program," NUWC-NPT Technical Document No. 11577, 25 October 2004.

${ }^{19}$ R. J. Urick, Principles of Underwater Sound, 3rd ed. (McGraw-Hill, New York, 1983), pp. 242, 42.

${ }^{20}$ H. Medwin and C. S. Clay, Fundamentals of Acoustical Oceanography (Academic Press, Boston, 1998), p. 417.

${ }^{21}$ K. G. Foote, "Correcting acoustic measurements of scatterer density for extinction,” J. Acoust. Soc. Am. 88, 1543-1546 (1990).

${ }^{22}$ T. K. Stanton, "Multiple scattering with applications to fish-echo processing," J. Acoust. Soc. Am. 73, 1164-1169 (1983).

${ }^{23}$ J. E. Ehrenberg, "A comparative analysis of in situ methods for directly measuring the acoustic target strength of individual fish," IEEE J. Oceanic Eng. 4, 141-152 (1979).

${ }^{24}$ M. B. Moffett and W. L. Konrad, "Nonlinear sources and receivers," in Encyclopedia of Acoustics, edited by M. J. Crocker (Wiley, New York, 1997), Vol. 1, pp. 607-617.

${ }^{25}$ M. B. Moffett and R. H. Mellen, "CONVOL: A computer program for parametric source nearfield and farfield beam patterns," NUSC Technical Memorandum, TM No. 791132, 17 July 1979.

${ }^{26}$ R. H. Mellen and M. B. Moffett, "A numerical method for calculating the nearfield of a parametric acoustic source," J. Acoust. Soc. Am. 63, 1622-1624 (1978).

${ }^{27}$ M. B. Moffett and R. H. Mellen, "Nearfield characteristics of parametric acoustic sources," J. Acoust. Soc. Am. 69, 404-409 (1981).

${ }^{28}$ M. B. Moffett and R. H. Mellen, "On parametric source aperture factors," J. Acoust. Soc. Am. 60, 581-583 (1976).

${ }^{29}$ M. B. Moffett and R. H. Mellen, "Model for parametric acoustic sources," J. Acoust. Soc. Am. 61, 325-337 (1977).

${ }^{30}$ M. B. Moffett, R. H. Mellen, and W. L. Konrad, "Parametric acoustic sources of rectangular aperture," J. Acoust. Soc. Am. 63, 1326-1331 (1978).

${ }^{31}$ K. V. Mackenzie, "Nine-term equation for sound speed in the oceans," J. Acoust. Soc. Am. 70, 807-812 (1981).

${ }^{32}$ R. E. Francois and G. R. Garrison, "Sound absorption based on ocean measurements. Part II. Boric acid contribution and equation for total absorption," J. Acoust. Soc. Am. 72, 1879-1890 (1982).

${ }^{33}$ H. O. Berktay, "Possible exploitation of non-linear acoustics in underwater transmitting applications," J. Sound Vib. 2, 435-461 (1965).

${ }^{34}$ J. Naze and S. Tjøtta, "Nonlinear interaction of two sound beams," J. Acoust. Soc. Am. 37, 174-175 (1965). 
${ }^{35}$ E. A. Zabolotskaya and S. I. Soluyan, "A possible approach to the amplification of sound waves," Sov. Phys. Acoust. 13, 254-256 (1967).

${ }^{36}$ M. F. Hamilton and F. H. Fenlon, "Parametric acoustic array formation in dispersive fluids," J. Acoust. Soc. Am. 76, 1474-1492 (1984).

${ }^{37}$ E. A. Zabolotskaya and S. I. Soluyan, "Emission of harmonic and combination-frequency waves by air bubbles," Sov. Phys. Acoust. 18, 396-398 (1973)

${ }^{38}$ M. S. Korman and R. T. Beyer, "Nonlinear scattering of crossed ultrasonic beams in the presence of turbulence in water. I. Experiment," J. Acoust. Soc. Am. 84, 339-349 (1988).

${ }^{39}$ M. S. Korman and R. T. Beyer, "Nonlinear scattering of crossed ultrasonic beams in the presence of turbulence in water. II. Theory," J. Acoust. Soc. Am. 85, 611-620 (1989).

${ }^{40}$ M. S. Korman, "Enhancement of the nonlinear scattering of crossed sound beams in the presence of turbulence due to a concentration of bubbles in the interaction region (A), "J. Acoust. Soc. Am. 76, S30 (1984).
${ }^{41}$ E. Hammerstad, "Sound levels from Kongsberg multibeams," EM Technical Note, 20 September 2005, http://www.km.kongsberg.com/ks/web/ nokbg0397.nsf/AllWeb/F9980522E6621E89C1257085002C0BE7/\$file/ EM_technical_note_web_SoundLevelsFromKongsbergMultibeams.pdf? OpenElement (Last viewed 27 January 2012).

${ }^{42}$ K. G. Foote, H. P. Knudsen, G. Vestnes, D. N. MacLennan, and E. J. Simmonds, "Calibration of acoustic instruments for fish density estimation: a practical guide," ICES Cooperative Research Report 144 (1987).

${ }^{43}$ K. G. Foote, D. T. I. Francis, and P. R. Atkins, "Calibration sphere for lowfrequency parametric sonars," J. Acoust. Soc. Am. 121, 1482-1490 (2007).

${ }^{44}$ K. Olsen, J. Angell, F. Pettersen, and A. Løvik, "Observed fish reactions to a surveying vessel with special reference to herring, cod, capelin and polar cod," FAO Fish. Rep. 300, 131-138 (1983).

${ }^{45}$ E. Ona, O. R. Godø, N. O. Handegard, V. Hjellvik, R. Patel, and G. Pedersen, "Silent research vessels are not quiet," J. Acoust. Soc. Am. 121, EL145-EL150 (2007). 